Advanced Studies in Theoretical Physics

Vol. 11, 2017, no. 8, $365-386$

HIKARI Ltd, www.m-hikari.com

https://doi.org/10.12988/astp.2017.7520

\title{
Quantum Mechanics of Rectilinear Orbits of the Coulomb-Kepler Problem
}

\author{
Alexander Rauh \\ Department of Physics, University of Oldenburg \\ D-26111 Oldenburg, Germany
}

Copyright (c) 2017 Alexander Rauh. This article is distributed under the Creative Commons Attribution License, which permits unrestricted use, distribution, and reproduction in any medium, provided the original work is properly cited.

\begin{abstract}
Based on coherent states of the reversed harmonic oscillator, a wave function is proposed with the following three properties: (1) The mean values of position, $\mathbf{r}_{0}$, and velocity, $\mathbf{v}_{0}$, are parallel to the $x$ axis; (2) The wave function is extended by a curve parameter $w \geq 0$ such that, as a function of $w$, the mean values $\mathbf{r}_{w}$ and $\mathbf{v}_{w}$ describe a rectilinear orbit along the $x$ axis with the mean initial values $\mathbf{r}_{0}$ and $\mathbf{v}_{0}$; (3) The mean energy is constant with respect to $w$ by the leading order of the perturbation parameter $\epsilon$. For comparison, the classical one-dimensional equation of motion is integrated. Exact analytical equivalence between classical and the $\epsilon=0$ quantum limit is found, both for positive and negative mean energy. The curve parameter $w$ is in 1-1 correspondence with time $t \geq 0$.
\end{abstract}

PACS: 03.65.Nk, 02.70.Wz

Keywords: Coherent states, Rutherford backscattering, KustaanheimoStiefel transformation

\section{Introduction}

In the previous two articles [11, 12], both elliptic, hyperbolic, and parabolic Kepler orbits were derived from quantum theory in the classical limit. These orbits share the common property that an apex exists where the velocity is 
normal to the vector with origin in the force center. Equivalently, the orbits are connected with non-zero angular momentum, which in the macroscopic limit is large in units of $\hbar$. The apex conveniently served as initial point which was built into the wave function through the scalar length values $r_{0}$ and $v_{0}$ of the mean initial position and velocity vectors.

Due to the zero angular momentum, the previous wave function cannot be used in the rectilinear case. Clearly, quantum mechanical fluctuations forbid to reduce the problem to a one-dimensional Hamiltonian.

We have to adjust the parameters of the wave function such that the mean initial position vector and the velocity are parallel, or anti-parallel:

$$
\left\langle\psi_{0} \mid \mathbf{r} \psi_{0}\right\rangle=\left\{X_{0}, 0,0\right\}, \quad(1 / m)\left\langle\psi_{0} \mid \mathbf{p} \psi_{0}\right\rangle=\left\{\sigma V_{0}, 0,0\right\}, \quad \sigma= \pm 1
$$

where $X_{0}>0, V_{0}>0$, and $\mathbf{r}$ and $\mathbf{p}$ denote the position and momentum operators. The mean initial point is assumed to be always on the same side of the force center. In addition, the sign of the potential, $\alpha / r$, has to be considered. As perturbation parameter, the number $\epsilon=\hbar /\left(m X_{0} V_{0}\right)$ will be used, which generally is small in macroscopic situations.

Let us briefly discuss the classical motion which was illustrated in [8], based on a reduced one-dimensional Hamiltonian with a regularized attractive potential. In the case of positive energy, the trajectories, of course, escape to infinity. However, the case $\sigma=-1$ is intriguing, since initially the mass point falls towards the singularity. In order to obtain continuous curves, the potential, $-|\alpha| / r$, was regularized in [8] as $-|\alpha| /\left(r+\eta^{2}\right)$ with the consequence that the mass point moves through the center, at a finite speed, and escapes to infinity on the other side of the force center.

In a quantum mechanical treatment, the singularity at $r=0$ is smoothed out by the finite extension of the wave function; regularization is not necessary.

The case of positive energy and a repellent potential corresponds to Rutherford scattering. According to Eq. (3-67) in [4], the connection between the impact parameter $s$ and the scattering angle $\Theta$ is given by

$$
s=\text { const. } \cot (\Theta / 2) \text {. }
$$

A rectilinear trajectory corresponds to zero impact parameter, $s=0$, which, hence, leads to the scattering angle $\Theta=\pi$, i.e. to backscattering. This is in agreement with the classical limit of the quantum mechanical treatment to follow, but at variance with the result in [8]. It should be remarked that the angle $\Theta$ refers to a coordinate system with origin in the force center which is non-inertial in general. But, as can be shown, zero impact parameter implies backscattering also in the laboratory system when the target mass is finite, see, e.g., Eq. (3-72) in [4]. Rutherford backscattering spectrometry (RBS) is a widely used analytical tool in the sciences [10]; for recent works see for instance: 
in solid state and surface physics $[16,5]$, in earth science [3], in astrophysics [6], or in archaeology [9]. As compared to scattering theory which is concerned with asymptotic states, here we start with a wave function which is prepared at time $t=0$ for a finite distance and can be continuously followed for $t>0$. If the potential is attractive, the particle comes close to the scattering center after finite time. Thus, mean square deviations can be determined near the center, in principle, whereas in the limit of infinite time, the asymptotic case, the mean square deviation of position diverges by quantum diffusion in the given case.

As previously [11, 12], the dynamics is described by a curve parameter $w \geq 0$ for positive energy and by $\tilde{w} \geq 0$ with $w=\mathbf{i} \tilde{w}$, if the mean energy is negative. The parameter $\tilde{w}$ corresponds to the eccentric anomaly in the case of elliptic orbits. In order to establish the 1-1 connection with time $t$, it is crucial to ensure that the mean energy does not depend on the curve parameter. To this end, an open variable $\gamma_{0}$ which is built into the wave function has to be properly disposed of; $\gamma_{0}$ may depend on the curve parameter, in principle.

The quantum regime with non-zero perturbation parameter, $\epsilon \neq 0$, will be investigated in a further study; first attempts indicate that finite order perturbation limits the definition domain of the curve parameter such that the trajectory does not reach the force center; a kind of "horror singularitis". We think that the situation can be clarified, if one succeeds to fulfill energy conservation rigorously, i.e., non-perturbatively.

The mean values are analytically calculated with the help of Mathematica [15]. The task amounts to carry out five-dimensional integrals. The primary outputs often consist of sums with more than hundred terms. To simplify the expressions, useful tools are available by Mathematica, but the final compact results are mostly achieved only with additional efforts by the author. How can one check the results? In the given case, the classical limit of the quantum mechanical calculations agrees with classical mechanics; the equivalence is analytically examined and verified in Appendix B. Furthermore, the relative mean square deviations, for instance $\left(\Delta v_{i}\right)^{2} /\left\langle v_{i}\right\rangle^{2}$, should be of order $\epsilon$ in macroscopic situations like celestial mechanics; otherwise the trajectories of the planets or artificial satellites would be observably blurred by quantum fluctuations. The application of this kind of macroscopic principle will facilitate the mean value calculation of the kinetic energy where, to leading order, $\left\langle v_{i}^{2}\right\rangle=\left\langle v_{i}\right\rangle^{2}[1+\mathcal{O}(\epsilon)], i=x, y, z$. This property was explicitly verified in previous work $[11,12]$ and is also proved for the given case in work supplemental to this article [17]. 


\section{Implementing initial conditions}

In analogy to $[11,12]$, we adopt a wave function $\Psi_{w}$, which lives in the Kustaanheimo-Stiefel (KS) [7] configuration space $\mathbf{u}=\left\{u_{1}, u_{2}, u_{3}, u_{4}\right\} \in \mathbf{R}^{4}$,

$$
\Psi_{w}(\Phi)=C \exp \left[\mathbf{a}(w) \cdot \mathbf{u}(\Phi)-\Gamma(w) u^{2} / 2\right], \quad 0 \leq \Phi<2 \pi, \quad \operatorname{Real}(\Gamma)>0
$$

where the parameter vector $\mathbf{a} \in \mathbf{C}^{4}$ is specified by the initial conditions, $C$ is the normalization constant, and $w$ is a curve parameter with $w=0$ corresponding to the initial time $t=0$. The function (2), essentially, is the fourfold product of a coherent state of the reversed harmonic oscillator [11, 14], i.e., an oscillator with imaginary frequency. The connection of the vector $\mathbf{u}$ with $3-\mathrm{D}$ polar coordinates $(r, \theta, \varphi)$ can be written as follows $[2,11]$ :

$$
\begin{array}{ll}
u_{1}=\sqrt{r} \cos (\theta / 2) \cos (\varphi-\Phi) ; & u_{2}=\sqrt{r} \cos (\theta / 2) \sin (\varphi-\Phi) ; \\
u_{3}=\sqrt{r} \sin (\theta / 2) \cos (\Phi) ; & u_{4}=\sqrt{r} \sin (\theta / 2) \sin (\Phi),
\end{array}
$$

where

$$
r>0, \quad 0<\theta<\pi, \quad 0 \leq \varphi<2 \pi, \quad \text { and } \quad 0 \leq \Phi<2 \pi
$$

The KS phase $\Phi$ describes the extension to the forth dimension. Eventually, in physical space, the wave function, $\psi_{w}$, is obtained by projecting out the phase $\Phi$ as

$$
\psi_{w}=\int_{0}^{2 \pi} \mathrm{d} \Phi \Psi_{w}(\Phi) .
$$

The initial conditions (1) are implemented through the following parameter components $a_{k}(0)$ :

$$
\begin{array}{ll}
a_{1}(0)=\rho_{0} \cos \left(\Phi_{0}\right)[1+\mathbf{i} \sigma \nu], & a_{2}(0)=\rho_{0} \sin \left(\Phi_{0}\right)[1+\mathbf{i} \sigma \nu], \\
a_{3}(0)=a_{1}(0), & a_{4}(0)=-a_{2}(0),
\end{array}
$$

with the phase $\Phi_{0} \in(0,2 \pi)$ and the number $\nu>0$ being free constants, and

$$
\rho_{0}=\Gamma_{0} \sqrt{r_{0} / 2}>0, \quad \Gamma_{0}=\Gamma(w=0)>0, \quad \sigma= \pm 1,
$$

where, in the classical limit, $r_{0}=X_{0}$ is the initial distance between the apex and the force center; quantum correction will give rise to a re-normalization of $r_{0}$ in terms of the physical initial value $X_{0}$.

The result (6) is verified later on by the general mean values of position and velocity as a function of $w \geq 0$; a constructive derivation works in analogy to Sec.V of [11]. Where does the initial parameter $V_{0}$ of the velocity enter? From (2) it is seen that the initial width of the wave function is characterized by $\Gamma_{0}$. So we introduce the dimensionless parameter

$$
\kappa:=r_{0} \Gamma_{0}
$$


As it turns out, $\kappa$ is fixed by the initial parameters $X_{0}$ and $V_{0}$; it is a large number in macroscopic cases. We also introduce the dimensionless velocity parameter $\zeta$ and the velocity constant $v_{0}$ as follows:

$$
\zeta=v_{0} / V_{0}, \quad v_{0}=\hbar /\left(2 m X_{0}\right), \quad\left\langle v_{x}\right\rangle(w=0)=\sigma V_{0}, \quad \sigma= \pm 1 .
$$

As will be seen, $\kappa$ is a unique function of $\zeta$ and, thus, of the initial magnitudes $X_{0}, V_{0}$. The classical limit is obtained for $\zeta \rightarrow 0$. For specifics of the dependence $\kappa=\kappa(\zeta)$, see Sec. IV.

The dependence on $w$ results from the evolution of the coherent oscillator states according to [11]. In the hyperbolic case, i.e., for positive mean energy, it is described with the help of the auxiliary functions

$$
f(w)=\cosh (w)-\mathbf{i} \gamma_{0} \sinh (w), \quad h(w)=\left[f(w) f^{*}(w)\right]^{-1}
$$

where the star means complex conjugation. The parameter $\gamma_{0}$ will be chosen in a later step by the condition that the mean energy is constant with respect to the curve parameter $w$. According to [11], the extension to parameters $w>0$ proceeds as $\mathbf{a}(0) \rightarrow \mathbf{a}(w)$ and $\Gamma_{0} \rightarrow \Gamma(w)$ with

$$
\mathbf{a}(w)=\mathbf{a}(0) / f^{*}(w) ; \quad \Gamma(w)=\Gamma_{0} h(w)\left[1-\mathbf{i} \sinh (2 w)\left(1+\gamma_{0}^{2}\right) /\left(2 \gamma_{0}\right)\right],
$$

where $\Gamma_{0}>0$ is fixed by the initial data through (8) and (9).

\section{Mean values}

Henceforth, we set the number $\nu=1$, for simplicity. At variance with previous calculations $[11,12,13]$, for use in a further study, we will calculate mean values exactly when possible, without resorting to an asymptotic approximation. However, in the given article, only the leading order with respect to $\kappa$ will be needed, which is equivalent to the limit $\epsilon \rightarrow 0$ with $\epsilon=1 / \kappa$. The mean value of an operator $O$ will be calculated via the $\mathbf{u}$ space, as is outlined in Sec. 4.3, Appendix B, and Appendix C of [12],

$$
\left\langle\psi_{w} \mid O \psi_{w}\right\rangle=\int_{0}^{2 \pi} \mathrm{d} \phi \int_{-\infty}^{\infty}\left(8 u^{2}\right) \mathrm{d} u_{1} \ldots \mathrm{d} u_{4} \Psi_{w}\left(\Phi^{\prime}\right)^{*}\left[O \Psi_{w}(\Phi)\right] \quad \phi=\Phi-\Phi^{\prime}
$$

where

$$
\Psi_{w}(\Phi)=C \exp \left[\mathbf{a}(w, \Phi) \cdot \mathbf{u}-\Gamma(w) u^{2} / 2\right], \quad \mathbf{u}=\mathbf{u}(\Phi=0)
$$

As compared to $[11,12]$, the parameter vector a now differs due to the new initial conditions. Using (6), (7), and (11), we write (setting $\nu=1$ )

$$
\begin{aligned}
\mathbf{a} & \equiv \mathbf{a}(w, \Phi)=\left[\rho_{0} / f^{*}(w)\right](1+\mathbf{i} \sigma)\{\cos (\Phi), \sin (\Phi), \cos (\Phi),-\sin (\Phi)\},(14) \\
\mathbf{a}^{*} & \equiv \mathbf{a}^{*}\left(w, \Phi^{\prime}\right)=\left[\rho_{0} / f(w)\right](1-\mathbf{i} \sigma)\left\{\cos \left(\Phi^{\prime}\right), \sin \left(\Phi^{\prime}\right), \cos \left(\Phi^{\prime}\right),-\sin \left(\Phi^{\prime}\right)\right\}
\end{aligned}
$$


and introduce the four-vector $\mathbf{A}$ :

$$
\mathbf{A}=\mathbf{a}^{*}\left(w, \Phi^{\prime}\right)+\mathbf{a}(w, \Phi)
$$

The arbitrary KS phase $\Phi_{0}$ which appears in $\mathbf{a}(0)$ has been set equal to zero, for simplicity. Later on, we will find that the $y$ and $z$ components of the mean values of position and velocity vanish identically. This is due to the following symmetry of the parameter vectors:

$$
a_{1}(w, \Phi)=a_{3}(w, \Phi), \quad a_{2}(w, \Phi)=-a_{4}(w, \Phi) ; \quad A_{1}=A_{3} \quad A_{2}=-A_{4}
$$

For the calculation of mean values, we will express the observables in terms of the 4-vector $\mathbf{u}$. It turns out that the observables do not depend on the KS phase $\Phi$, see (3). As a consequence, the mean values of the position and velocity depend on the difference $\phi:=\Phi-\Phi^{\prime}$, only. From [12], we take over the dimensionless functions $\kappa_{0}$ and $\kappa_{1}$, which are modified by the new parameter vector a. They are defined through

$$
\mathbf{A} \cdot \mathbf{A}=4 r_{0} \Gamma_{R}^{2}\left(\kappa_{0}+\kappa_{1} \cos (\phi)\right), \quad \phi=\Phi-\Phi^{\prime},
$$

and now read (setting $\nu=1$ )

$$
\begin{aligned}
& \kappa_{0}=\gamma_{0} \sigma \sinh (2 w) \\
& \kappa_{1}=h^{-1}(w)=\cosh ^{2}(w)+\gamma_{0}^{2} \sinh ^{2}(w) .
\end{aligned}
$$

\subsection{Normalization}

The normalization condition is worked out in Appendix A1 with the result

$$
\begin{aligned}
1 \equiv & 2\left(2 \pi r_{0} / K\right)^{3} \exp \left(K \kappa_{0}\right) I_{0}(\kappa) C^{2}\left[\kappa I_{\kappa}+2+K \gamma_{0} \sigma \sinh (2 w)\right] \\
& K=r_{0} \Gamma_{R}=\kappa h(w), \quad \kappa=r_{0} \Gamma_{0}
\end{aligned}
$$

where we introduced the quotient of the modified Bessel functions $I_{1}$ and $I_{0}$ :

$$
I_{\kappa}=I_{1}(\kappa) / I_{0}(\kappa)
$$

We will use repeatedly the following properties of the dynamic magnitude parameter $K$ :

$$
\begin{aligned}
K / \kappa=h(w) & =\left[\cosh ^{2}(w)+\gamma_{0}^{2} \sinh ^{2}(w)\right]^{-1} \\
& =2\left[1-\gamma_{0}^{2}+\left(1+\gamma_{0}^{2}\right) \cosh (2 w)\right]^{-1}
\end{aligned}
$$




\subsection{Reciprocal distance}

The calculation of $\langle 1 / r\rangle$ is facilitated by the fact that the observable $1 / r \equiv 1 / u^{2}$ cancels against the metric factor $u^{2}$, so one needs no parameter differentiation with respect to $\Gamma_{R}$. One obtains straightforwardly

$$
\begin{aligned}
\langle 1 / r\rangle & =\left(K / r_{0}\right)\left[2+\kappa I_{\kappa}+K \gamma_{0} \sigma \sinh (2 w)\right]^{-1} \\
& =\left(K / X_{0}\right)\left(\kappa+3 I_{\kappa}\right)\left[\left(2+\kappa I_{\kappa}\right)\left(2+\kappa I_{\kappa}+K \gamma_{0} \sigma \sinh (2 w)\right)\right]^{-1}
\end{aligned}
$$

where in the last equation we used the renormalization of $r_{0}$ in terms of $X_{0}$, see $(34)$ below. To leading order in $\kappa$, we obtain with the aid of the property (22) for $K$ :

$$
\langle 1 / r\rangle=X_{0}^{-1}\left[\cosh (w)+\gamma_{0} \sigma \sinh (w)\right]^{-2}+\mathcal{O}(1 / \kappa)
$$

\subsection{Position vector}

In terms of the vector $\mathbf{u}$, the cartesian position components are defined as

$$
x=2\left(u_{1} u_{3}-u_{2} u_{4}\right), \quad y=2\left(u_{1} u_{4}+u_{2} u_{3}\right), \quad z=u_{1}^{2}+u_{2}^{2}-u_{3}^{2}-u_{4}^{2} .
$$

The calculation of the mean values is sketched in Appendix A2; it is closely based on the method outlined in Appendix B of [12]. We use the abbreviation

$$
F(\mathbf{A}):=A^{2}+16 \Gamma_{R}
$$

and obtain for the components $x_{j}$ with $x_{1} \equiv x, x_{2} \equiv y, x_{3} \equiv z$

$$
\left\langle x_{j}\right\rangle=\frac{\pi^{2} C^{2}}{\Gamma_{R}^{6}} \int_{0}^{2 \pi} \mathrm{d} \phi F(\mathbf{A}) F_{j}(\mathbf{A}) \exp \left[K\left(\kappa_{0}+\kappa_{1}\right)\right],
$$

where

$$
F_{x}=A_{1} A_{3}-A_{2} A_{4}, \quad F_{y}=A_{2} A_{3}+A_{1} A_{4}, \quad F_{z}=A_{1}^{2}+A_{2}^{2}-A_{3}^{2}-A_{4}^{2} .
$$

By the symmetry (16), the $y$ and $z$ components vanish identically:

$$
F_{y}=F_{z}=0, \quad \text { and } \quad\langle y\rangle=\langle z\rangle=0
$$

As is shown in Appendix A2, the non-zero component has the mean value

$$
\langle x\rangle=\pi\left(4 \pi r_{0}^{2} / K\right)^{2} K^{-1} C^{2} \exp \left(K \kappa_{0}\right) I_{0}(\kappa)\left[c_{0}+c_{1} I_{\kappa}+c_{2}\left(-I_{\kappa} / \kappa+1\right)\right],
$$

with

$$
c_{0}=\kappa_{0}\left(4+K \kappa_{0}\right), \quad c_{1}=2 \kappa \kappa_{0}+4 \kappa_{1}, \quad c_{2}=\kappa \kappa_{1},
$$


where we used the relation $K \kappa_{1}=\kappa$. After inserting the c coefficients, we arrive at

$\langle x\rangle=\pi\left(4 \pi r_{0}^{2} / K\right)^{2} K^{-1} C^{2} \exp \left(K \kappa_{0}\right) I_{0}(\kappa)\left[4 \kappa_{0}+K \kappa_{0}^{2}+\kappa \kappa_{1}+\left(3 \kappa_{1}+2 \kappa \kappa_{0}\right) I_{\kappa}\right]$.

In order to eliminate the normalization constant $C$, we divide (32) by the condition (20) to obtain

$$
\langle x\rangle=r_{0} \frac{4 \kappa_{0}+K \kappa_{0}^{2}+\kappa \kappa_{1}+\left(3 \kappa_{1}+2 \kappa \kappa_{0}\right) I_{\kappa}}{\kappa I_{\kappa}+2+K \gamma_{0} \sigma \sinh (2 w) .}
$$

The initial value $X_{0}=\langle x\rangle(w=0)$ is obtained by making use of $K(0)=\kappa$, $\kappa_{0}(0)=0$, and $\kappa_{1}(0)=1$. We find the following connection between the wave function parameter $r_{0}$ and the mean initial component $X_{0}$ :

$$
X_{0}=r_{0} \frac{\kappa+3 I_{\kappa}}{2+\kappa I_{\kappa}} \quad \text { implying } \quad r_{0}=X_{0} \frac{2+\kappa I_{\kappa}}{\kappa+3 I_{\kappa}} .
$$

To leading order with respect to $\kappa$ (or $K=\kappa h(w)$ ), we find

$$
\langle x\rangle=X_{0}\left[\cosh (w)+\gamma_{0} \sigma \sinh (w)\right]^{2}[1+\mathcal{O}(1 / \kappa)] .
$$

\subsection{Velocity}

As it is sketched in Appendix A3, the following intermediate expressions are obtained for the velocity components $v_{j}$ :

$$
\left\langle v_{j}\right\rangle=-\frac{16 \pi^{2} \mathbf{i} \hbar C^{2}}{m \Gamma_{R}^{4}} \int_{0}^{2 \pi} \mathrm{d} \phi G_{j}(\mathbf{a}, \mathbf{A}) \exp \left[K\left(\kappa_{0}+\kappa_{1} \cos (\phi)\right)\right], \quad j=x, y, z,
$$

where

$$
\begin{aligned}
G_{x}= & \mathbf{i} \Gamma_{I}\left(A_{2} A_{4}-A_{1} A_{3}\right)+ \\
& \Gamma_{R}\left[A_{1} a_{3}+A_{3} a_{1}-A_{2} a_{4}-A_{4} a_{2}-A_{1} A_{3}+A_{2} A_{4}\right] \\
G_{y}= & -\mathbf{i} \Gamma_{I}\left(A_{2} A_{3}+A_{1} A_{4}\right)+ \\
& \Gamma_{R}\left[A_{2} a_{3}+A_{3} a_{2}+A_{1} a_{4}+A_{4} a_{1}-A_{2} A_{3}-A_{1} A_{4}\right] \\
G_{z}= & -(1 / 2) \mathbf{i} \Gamma_{I}\left(A_{1}^{2}+A_{2}^{2}-A_{3}^{2}-A_{4}^{2}\right)+ \\
& (1 / 2) \Gamma_{R}\left[A_{3}^{3}+A_{4}^{2}-A_{1}^{2}-A_{2}^{2}+2\left(A_{1} a_{1}+A_{2} a_{2}-A_{3} a_{3}-A_{4} a_{4}\right)\right] .
\end{aligned}
$$

By the symmetry (16), the $y$ and $z$ components vanish identically:

$$
G_{y}=G_{z}=0, \quad \text { and } \quad\left\langle v_{y}\right\rangle=\left\langle v_{z}\right\rangle=0 .
$$

For the $x$ component one finds, see Appendix A3.,

$$
\left\langle v_{x}\right\rangle=4 \pi^{3}\left(C^{2} \hbar r_{0}^{2} / m\right)\left(K^{2} \gamma_{0}\right)^{-1} \kappa I_{0}(\kappa)\left[2 \gamma_{0} \sigma \cosh (2 w)+\left(1+\gamma_{0}^{2}\right) \sinh (2 w) I_{\kappa}\right],
$$




\begin{tabular}{c|ccc}
\hline \hline model & $\begin{array}{c}\text { electron } \\
\text { proton }\end{array}$ & $\begin{array}{c}\text { proton } \\
\text { proton }\end{array}$ & $\begin{array}{l}\text { Satellite } \\
\text { Earth }\end{array}$ \\
\hline$X_{0}$ & $10^{-10} \mathrm{~m}$ & $10^{-10} \mathrm{~m}$ & $4 \times 10^{7} \mathrm{~m}$ \\
$V_{0}$ & $16 \mathrm{eV}$ & $10 \mathrm{eV}$ & $5000 \mathrm{~m} / \mathrm{s}$ \\
$\mathrm{m}$ & $m_{e}$ & $m_{p}$ & $m_{S}=1000 \mathrm{~kg}$ \\
$\alpha$ & $-\frac{e^{2}}{4 \pi \epsilon_{0}}$ & $+\frac{e^{2}}{4 \pi \epsilon_{0}}$ & $-G m_{S} m_{E}$ \\
$\zeta$ & 4.0986 & 138.87 & $4 \times 10^{48}$ \\
$\kappa$ & 4.5284 & 138.87 & $\kappa=\zeta$ \\
$\lambda=\gamma_{0}$ & 3.1618 & 0.6460 & 2.2247 \\
\hline \hline
\end{tabular}

Table 1: Three examples for a rectilinear motion (head-on collision with $\sigma=$ -1 ) for mean initial distance and speed $X_{0}$ and $V_{0}$. The meaning of the symbols is: $e$ proton charge, $m_{e(p)}$ electron (proton) mass, $\alpha$ the coupling constant, $\epsilon_{0}$ electric constant, $\zeta=V_{0}$ in units of $v_{0}=\hbar /\left(2 m X_{0}\right), \kappa$ the width parameter of the wave function as calculated from $\zeta, \lambda^{2}$ the quotient of the initial kinetic energy and initial total energy, $\gamma_{0}$ a wave function parameter calculated from energy conservation. To leading order in $\kappa, \gamma_{0}$ equals $\lambda$. It is noticed that the mean total energy is positive in the three models which implies that $\lambda$ is real.

and after eliminating the normalization constant $C$

$$
\left\langle v_{x}\right\rangle=\frac{\hbar K \kappa}{4 m r_{0} \gamma_{0}} \frac{2 \gamma_{0} \sigma \cosh (2 w)+\left(1+\gamma_{0}^{2}\right) \sinh (2 w) I_{\kappa}}{2+\kappa I_{\kappa}+K \gamma_{0} \sigma \sinh (2 w)},
$$

where $r_{0}$ has to be replaced by (34) in terms of the mean initial value $X_{0}$.

The initial velocity, $U_{0}=\sigma V_{0}$, at $w=0$ (remember $\left.K(0)=\kappa h(0)=\kappa\right)$ comes out as

$$
V_{0}=v_{0} \kappa^{2}\left(3 I_{\kappa}+\kappa\right) /\left(2+\kappa I_{\kappa}\right)^{2}, \quad v_{0}=\hbar /\left(2 m X_{0}\right) .
$$

To leading order in $\kappa$, we find

$$
\left\langle v_{x}\right\rangle=\frac{\hbar \kappa}{2 m X_{0} \gamma_{0}} \frac{\gamma_{0} \sigma \cosh (w)+\sinh (w)}{\cosh (w)+\gamma_{0} \sigma \sinh (w)}, \quad \sigma= \pm 1 .
$$

\section{Width of the wave packet}

The width parameter $\kappa$ will be determined in terms of the initial data.

Before, let us comment on Tab. I by specifying the coupling constant $\alpha$ of the Coulomb-Kepler problem. In the electric case the coupling reads

$$
\alpha_{e l}=1 /\left(4 \pi \epsilon_{0}\right) Q_{1} Q_{2},
$$

where $\epsilon_{0}$ is the electric constant, and $Q_{1}$ and $Q_{2}$ are electric charges of positive or negative sign. In the case of two classically interacting protons, the quotient 
of the gravitational coupling constant $\alpha_{G}=-G M_{1} M_{2}$ and of $\alpha_{e l}$ has the order of magnitude

$$
\left|\alpha_{G} / \alpha_{e l}\right| \approx 10^{-36}
$$

a well known example for the relative weakness of gravitation.

In the following, we determine $\kappa$ in terms of the initial values $X_{0}, V_{0}$. The function $I_{\kappa}$, defined in (21), can be numerically evaluated by calling the functions BesselI $[1, \kappa]$ and BesselI $[0, \kappa]$ of Mathematica [15], which, however, exponentially increase with $\kappa$. For large values of $\kappa$, one better uses the asymptotic expressions of the modified Bessel functions, see formula (9.7.1) in [1], and takes advantage of the fact that the exponential factors $\exp (\kappa)$ of the asymptotic expressions drop out in $I_{\kappa}$. For $\kappa=\{5,10,100\}$ the following asymptotic approximation has errors of the order $\left\{10^{-4}, 10^{-6}, 10^{-12}\right\}$ :

$$
I_{\kappa}^{(5)}=1-\epsilon / 2-\epsilon^{2} / 8-\epsilon^{3} / 8-(25 / 128) \epsilon^{4}-(13 / 32) \epsilon^{5}, \quad \epsilon=1 / \kappa .
$$

As can be proved from the integral representation (9.6.19) [1] of modified Bessel functions, one has the rigorous bounds $0 \leq I_{\kappa}<1$ for $\kappa \geq 0$ with the limit $I_{\infty}=1$. For the relation $\kappa=\kappa(\zeta)$, one solves (41) for $\kappa$ in terms of $\zeta=V_{0} / v_{0} \equiv 2 m X_{0} V_{0} / \hbar$ :

$$
\zeta=\kappa^{2}\left(3 I_{\kappa}+\kappa\right) /\left(2+\kappa I_{\kappa}\right)^{2}, \quad 0 \leq I_{\kappa}<1
$$

By (46) and through $\zeta$, the width parameter $\kappa$ of the wave function is implicitely defined in terms of the initial data. Large values of $\zeta$ imply large values of $\kappa$. With the aid of (45), we derive the asymptotic approximation

$$
\kappa^{(4)}=\zeta+(7 / 2) \eta-(43 / 4) \eta^{2}+(101 / 8) \eta^{3}+(1957 / 32) \eta^{4}, \quad \eta=1 / \zeta .
$$

The relative errors for $\zeta=\{5,10,100\}$ are of the order $\Delta \kappa \equiv \kappa-\kappa^{(4)} \approx$ $\left\{2 \times 10^{-2}, 3 \times 10^{-4}, 4 \times 10^{-10}\right\}$.

\section{Energy conservation}

In [12], where the mean initial velocity was orthogonal to the mean initial position vector, the parameter $\gamma_{0}$ was related to the orbit eccentricity $e$ with $\gamma_{0}^{2}=(e+1)\left[\nu^{2}(e-1)\right]^{-1}$ for $e>1$. The crucial point was the requirement of energy conservation with respect to $w$. In the following, we will fix $\gamma_{0}$ from energy conservation to leading order in $\kappa$. Principally, $\gamma_{0}$ could turn out as $w$-dependent. But to our surprise, the parameter comes out being constant to leading and next higher (not shown in this article) order in $\kappa$.

Clearly, $E \equiv\langle H\rangle$ is constant in time provided that the initial state evolves by the unitary operator $\exp [-\mathbf{i} H t / \hbar]$. Since we will assume that time dependence of the wave function enters exclusively through the curve parameter 
$w=w(t)$, we require that the mean energy does not depend on the curve parameter $w$ in some interval $0 \leq w<w_{1}$.

We have to determine the following mean value:

$$
E:=\left\langle\psi_{w} \mid H \psi_{w}\right\rangle, \quad H=\frac{m}{2}\left(v_{x}^{2}+v_{y}^{2}+v_{z}^{2}\right)+\frac{\alpha}{r} .
$$

From the mean value of $\left\langle v_{x}\right\rangle$, see (42), it is seen that, to leading order, $E$ is proportional to $\kappa^{2}$, which implies the perturbative ansatz

$$
E=\kappa^{2} E_{0}(w)+\mathcal{O}(\kappa)
$$

By the "macroscopic principle" explained in the Introduction, the calculation of $\left\langle v_{i}^{2}\right\rangle$ is reduced to the the mean values of $v_{i}$, i.e., to leading order in $\kappa$, we can use the relations

$$
\left\langle v_{x}^{2}\right\rangle=\left\langle v_{x}\right\rangle^{2}, \quad\left\langle v_{y, z}^{2}\right\rangle=\left\langle v_{y, z}\right\rangle^{2}=0 .
$$

Thus, we obtain with the aid of (42) and (24)

$$
\begin{aligned}
\kappa^{2} E_{0}(w)= & \frac{\hbar^{2} \kappa^{2}}{8 m X_{0}^{2} \gamma_{0}^{2}}\left(\frac{\gamma_{0} \sigma \cosh (w)+\sinh (w)}{\cosh (w)+\gamma_{0} \sigma \sinh (w)}\right)^{2}+ \\
& \left(\alpha / X_{0}\right)\left[\cosh (w)+\gamma_{0} \sigma \sinh (w)\right]^{-2}
\end{aligned}
$$

We require that $\Delta E_{0}$ does not depend on $w$ :

$$
\begin{aligned}
\Delta E_{0}: & =\kappa^{2}\left[E_{0}(w)-E_{0}(0)\right]=E_{N} / E_{D} \\
E_{N} & =(1 / 2) G_{1}\left[-\left(1+\gamma_{0}^{2}\right)+\left(1+\gamma_{0}^{2}\right) \cosh [2 w]+2 \gamma_{0} \sigma \sinh (2 w)\right], \\
E_{D} & =8 m X_{0}^{2} \gamma_{0}^{2}\left[\cosh (w)+\gamma_{0} \sigma \sinh (w)\right]^{2}, \\
G_{1} & =-8 m X_{0} \alpha \gamma_{0}^{2}-\hbar^{2} \kappa^{2}\left(\gamma_{0}^{2}-1\right) .
\end{aligned}
$$

Obviously, $\Delta E_{0}=0$, if $G_{1}=0$ which, with the scaling $\alpha \rightarrow \beta$ and $\alpha=\kappa^{2} \beta$, implies

$$
\gamma_{0}^{2} \equiv \lambda^{2}=\hbar^{2} \kappa^{2} /\left(\hbar^{2} \kappa^{2}+8 m X_{0} \alpha\right)=\hbar^{2} /\left(\hbar^{2}+8 m X_{0} \beta\right)
$$

By (47), we can express $\hbar \kappa$ in terms of the initial position and speed $X_{0}$ and $V_{0}$, respectively, which is to leading order

$$
\hbar \kappa=\hbar \zeta=2 m X_{0} V_{0}
$$

with the consequence that

$$
\lambda^{2}=\frac{(m / 2) V_{0}^{2}}{(m / 2) V_{0}^{2}+\alpha / X_{0}}=\frac{E_{k i n}^{(0)}}{E_{0}}
$$


which is the quotient of the initial kinetic energy $E_{k i n}^{(0)}$ and the initial total energy $E_{0}$. If $E_{0} \geq 0$, then $\lambda^{2}>0$; else $\lambda$ is purely imaginary and requires that the curve parameter $w$ has to be analytically continued to purely imaginary values , $w \rightarrow \mathbf{i} \tilde{w}$, as in the case of elliptic orbits [12].

For negative mean energy, in order that $\langle x\rangle$ initially increases for $\tilde{w} \geq 0$ and $\sigma=+1$, one has to choose the sign of $\sqrt{\lambda^{2}} \equiv \sqrt{-\tilde{\lambda}^{2}}$ as follows:

$$
\gamma_{0}=-\mathbf{i} \tilde{\lambda}, \quad \tilde{\lambda}^{2}=m V_{0}^{2} /\left(2\left|E_{0}\right|\right), \quad E_{0}<0, \quad \tilde{\lambda}>0 .
$$

We obtain from (35) and (42) to leading order in $\kappa$

$$
\begin{aligned}
\langle x\rangle & =X_{0}[\cos (\tilde{w})+\tilde{\lambda} \sigma \sin (\tilde{w})]^{2}, \\
\left\langle v_{x}\right\rangle & =\frac{V_{0}}{\tilde{\lambda}} \frac{\tilde{\lambda} \sigma \cos (\tilde{w})-\sin (\tilde{w})}{\cos (\tilde{w})+\tilde{\lambda} \sigma \sin (\tilde{w})}, \quad \tilde{\lambda}>0 .
\end{aligned}
$$

As it is observed from the leading order expressions (57) and (58), $\langle x\rangle \geq 0$, and when $\langle x\rangle=0$, which is the singular center, then $\left\langle v_{x}\right\rangle=\infty$ and changes sign. This describes an oscillating mean motion between the maximal elongation $\langle x\rangle_{\max }=\left(1+\tilde{\lambda}^{2}\right) X_{0}$ and the force center, where the mean trajectory is reflected.

\section{Time dependence}

For the given Hamiltonian, the commutator relation $\mathrm{d} x /(\mathrm{d} t) \equiv(\mathbf{i} / \hbar)[H, x]=$ $p_{x} / m=v_{x}$ implies the necessary condition

$$
\mathrm{d}\langle x\rangle /(\mathrm{d} t)=\left\langle v_{x}\right\rangle
$$

which is true for any initial state which evolves by the operator $\exp [-\mathbf{i} H t / \hbar]$. As a consequence of (59), one obtains the following first order differential equation for $w(t)$ :

$$
\partial_{t} w \equiv F(w)=\left\langle v_{x}\right\rangle /\left(\partial_{w}\langle x\rangle\right) .
$$

In order that $w$ and $t$ are in 1-1 relation, we require that

$$
\partial_{t} w>0 \text { for } w \in\left(0, w_{c}\right), \quad w_{c}>0 .
$$

The critical interval limit, $w_{c}$, is determined by the property that $\partial_{t} w$ changes sign the first time for $w>0$. With the aid of (54), one derives from (60) for positive mean energy, using (35) and (42), and for negative energy, using (57) and (58), to leading order in $\kappa$,

$$
\begin{aligned}
& \partial_{t} w=\frac{V_{0}}{2 X_{0} \lambda}[\cosh (w)+\lambda \sigma \sinh (w)]^{-2}>0, \quad E_{0}>0 \\
& \partial_{t} \tilde{w}=\frac{V_{0}}{2 X_{0} \tilde{\lambda}}[\cos (\tilde{w})+\tilde{\lambda} \sigma \sin (\tilde{w})]^{-2}>0, \quad E_{0}<0
\end{aligned}
$$


which tells that the curve parameters $w$ and $\tilde{w}$ are in 1-1 correspondence with $t$ for all $t \geq 0$.

It is convenient to introduce the time constant $T_{0}$,

$$
T_{0}^{2}=m X_{0}^{2} /\left(2\left|E_{0}\right|\right)=\left(X_{0} / V_{0}\right)^{2}|\lambda|^{2},
$$

together with the dimensionless length, time, and velocity magnitudes $\xi, \tau$, and $\eta_{x}$, respectively, which are defined as

$$
x=X_{0} \xi, \quad t=T_{0} \tau, \quad v_{x}=\left(X_{0} / T_{0}\right) \eta_{x} \equiv\left(V_{0} /|\lambda|\right) \eta_{x}
$$

In the case $E_{0}>0$, the following dimensionless mean values result from (35) and (42) to leading order in $\kappa$

$$
\begin{aligned}
\langle\xi\rangle & =[\cosh (w)+\lambda \sigma \sinh (w)]^{2} \\
\left\langle\eta_{x}\right\rangle & =\frac{\lambda \sigma \cosh (w)+\sinh (w)}{\cosh (w)+\lambda \sigma \sinh (w)}, \quad E_{0}>0 .
\end{aligned}
$$

For negative mean energy, one writes (57) and (58) in the dimensionless forms

$$
\begin{aligned}
\langle\xi\rangle & =[\cos (\tilde{w})+\tilde{\lambda} \sigma \sin (\tilde{w})]^{2} \\
\left\langle\eta_{x}\right\rangle & =\frac{\tilde{\lambda} \sigma \cos (\tilde{w})-\sin (\tilde{w})}{\cos (\tilde{w})+\tilde{\lambda} \sigma \sin (\tilde{w})}, \quad E_{0}<0 .
\end{aligned}
$$

\subsection{Positive mean energy}

From (60) and (66), and using the relation $T_{0}=X_{0} \lambda / V_{0}$, we obtain

$$
w^{\prime}(\tau)=(1 / 2)[\cosh (w)+\sigma \lambda \sinh (w)]^{-2} .
$$

Integration proceeds by separation of variables. For the initial condition $w(0)=0$, we obtain the following analog to Kepler's equation:

$$
\tau=w\left(1-\lambda^{2}\right)+2 \sigma \lambda \sinh ^{2}(w)+\left(1+\lambda^{2}\right) \cosh (w) \sinh (w), \quad \lambda>0 .
$$

It should be noticed that for positive mean energy $\lambda>1$, if the potential is attractive, otherwise, in the repellent case, $0<\lambda<1$.

In order that $w$ is a proper curve parameter, it should be in one to one correspondence with time $\tau$. This is the case for $w \geq 0$, since by $(68) w^{\prime}(\tau)>0$ for $w \geq 0$ both for $\sigma=1$ and $\sigma=-1$. The case $\sigma=1$ is physically obvious, since the initial speed is in the opposite direction to the force center, so the mass point monotonically escapes to infinity. In the case $\sigma=-1$, the mass point starts to move towards the force center at $x=0$, where, if the potential is attractive, $w=w_{s}=\tanh ^{-1}(1 / \lambda)$. The velocity at $w_{s}$ is infinite, and there 
is backscattering with the velocity, given in (66b), reversed and the mass point continues to move towards an arbitrarily large distance $x>0$. From (69), we infer the scaled time $\tau_{s}$ elapsed between start and the instance of back scattering, this means the time which corresponds to the interval $w \in\left(0, w_{s}\right)$. With the aid of the formulas,

$$
\cosh \left[\tanh ^{-1}(1 / \lambda)\right]=\lambda\left(\lambda^{2}-1\right)^{-1 / 2}, \quad \sinh \left[\tanh ^{-1}(1 / \lambda)\right]=\left(\lambda^{2}-1\right)^{-1 / 2},
$$

one finds for an attractive potential with $\lambda>1$

$$
\tau_{s}=\lambda+\left(1-\lambda^{2}\right) \tanh ^{-1}(1 / \lambda), \quad \lambda^{2} \equiv m V_{0}^{2} /\left(2 E_{0}\right)>1, \quad \sigma=-1 .
$$

\subsection{Negative mean energy}

The case $E_{0}<0$ is possible only for an attractive potential. From (60) and (67), we infer

$$
\tilde{w}^{\prime}(\tau)=(1 / 2)[\cos (\tilde{w})+\sigma \tilde{\lambda} \sin (\tilde{w})]^{-2}, \quad \tilde{\lambda}^{2}=m V_{0}^{2} /\left(2\left|E_{0}\right|\right)>0 .
$$

Integration leads to the following analog to Kepler's equation:

$$
\tau=\tilde{w}\left(1+\tilde{\lambda}^{2}\right)+\left(1-\tilde{\lambda}^{2}\right) \cos (\tilde{w}) \sin (\tilde{w})+2 \sigma \tilde{\lambda} \sin ^{2}(\tilde{w}), \quad \tilde{w} \geq 0 .
$$

The instances of hitting the singularity are at

$$
\begin{aligned}
& 0<w_{+}=-\arctan (1 / \tilde{\lambda})+n \pi, \quad n=1,2, \ldots \quad \text { if } \quad \sigma=1, \\
& 0<w_{-}=\arctan (1 / \tilde{\lambda})+n \pi, \quad n=0,1, \ldots \quad \text { if } \quad \sigma=-1 .
\end{aligned}
$$

There is periodic bouncing back at $\xi=0$ with the period $\Delta w=\pi$, which corresponds to the time period

$$
\Delta T=\pi T_{0}\left(1+\tilde{\lambda}^{2}\right)
$$

independent of $\sigma= \pm 1$. By $(72), \tilde{w}^{\prime}(\tau)>0$ which guarantees that $\tau$ and $\tilde{w}$ are $1-1$ correspondence for $\tilde{w} \geq 0$, this means over arbitrary many periods.

\section{Comparison with classical mechanics}

We solve the classical one-dimensional equation of motion for the rectilinear case without the attempt of regularizing the Coulomb potential. At the branch points the solution will be ambiguous which is in contrast to the classical limit of the quantum theory presented above. Due to the branch points, we will have to distinguish quite some special cases. 
We start from energy conservation

$$
\frac{m}{2}\left(\frac{\mathrm{d} x}{\mathrm{~d} t}\right)^{2}+\frac{\alpha}{x}=E_{0},
$$

where $E_{0}$ is the initial energy, a first integration constant. Note that $\alpha$ is negative in the case of an attracting interaction. In terms of the dimensionless magnitudes defined in (64) and (65), one obtains

$$
\left(\frac{\mathrm{d} \xi}{\mathrm{d} \tau}\right)^{2}+\frac{\mu}{\xi}=\eta, \quad \eta= \pm 1,
$$

where

$$
\mu=\alpha /\left(X_{0}\left|E_{0}\right|\right), \quad \eta=\operatorname{sign}\left(E_{0}\right)= \pm 1 .
$$

From (78), we infer the first order differential equation

$$
\frac{\mathrm{d} \xi}{\mathrm{d} \tau}=\sigma \sqrt{\frac{\eta \xi-\mu}{\xi}}
$$

with the initial initial conditions

$$
\xi(0)=1, \quad[\mathrm{~d} \xi /(\mathrm{d} \tau)]_{\tau=0}=\sigma|\lambda|, \quad \sigma= \pm 1
$$

where the following connection between $\lambda$ and $\mu$ was used:

$$
|\lambda|^{2}+\mu=\eta \equiv \operatorname{sign}\left(E_{0}\right)
$$

We assume the initial point at a positive coordinate always and restrict to intervals which do not contain the singular point $\xi=0$ or turning points (if the energy $E_{0}$ is negative). Since $\left(\mathrm{d} \xi /(\mathrm{d} \tau)^{2} \geq 0\right.$, we have the inequalities

$$
(i): \eta \xi-\mu>0 ; \quad(i i): \xi>0 ; \quad(i i i): \mathrm{d} \tau>0
$$

where (iii) implies motion in forward time. The integration of (80) is elementary by separation of variables. Implementing the initial condition $\xi=1$ at $\tau=0$, we obtain

$$
\begin{aligned}
\tau(\xi)= & \sigma\left[\sqrt{\xi(\xi-\mu)}-\lambda+\mu \ln \left(\frac{\sqrt{\xi}+\sqrt{\xi-\mu}}{1+\lambda}\right)\right], \quad \mu=1-\lambda^{2} \\
& \xi \geq 1 \quad \text { for } \quad \sigma=1 ; \quad \xi_{0}<\xi \leq 1 \quad \text { for } \quad \sigma=-1, \quad E_{0}>0
\end{aligned}
$$

where the lower boundary $\xi_{0}=0$, if the potential is attractive with $\lambda^{2}>1$, else $\xi_{0}=1-\lambda^{2}$, if the potential is repellent with $\lambda^{2}<1$. For negative mean 
energy, we obtain

$$
\begin{aligned}
\tau(\xi)= & \sigma\left\{\tilde{\lambda}-\sqrt{\xi(-\xi-\mu)}-\mu\left[\arctan \left(\sqrt{\frac{\xi}{-\xi-\mu}}\right)-\right.\right. \\
& \left.\left.\arctan \left(\frac{1}{\tilde{\lambda}}\right)\right]\right\}, \quad E_{0}<0, \quad \mu=-1-\tilde{\lambda}^{2} ; \\
& 1 \leq \xi<1+\tilde{\lambda}^{2} \text { for } \sigma=1 ; \quad 0<\xi \leq 1 \text { for } \sigma=-1 .
\end{aligned}
$$

The results for $\tau(\xi)$ can be easily verified by differentiation of (84) and (85) with respect to $\tau$ and by taking into account (80).

As to the definition domains, some comments are in order. The functions above have branch points which reflect the $1 / r$ singularity at $\xi=0$ and the turning points where the velocity is zero in the case of negative energy. The definition domains are restricted above to the basic intervals without the attempt of analytic continuation beyond the branch points.

If $\sigma=1$ and $E_{0}>0$, then, by (66a), the coordinate $\xi$, and by (85), the time $\tau$ monotonously increase towards infinity, whereas for $\sigma=-1$ the initial motion is towards the singularity at $\xi=0$; in order to describe backscattering, one would have to analytically continue properly at the branch point $\xi=0$. In the case of negative energy $E_{0}<0$, one also would have to properly deal with the branching at the turning point in order to describe periodic oscillation.

For comparison, the parametric representation by the quantum mechanical treatment above avoids the complication with branch-points; it continuously describes the complete trajectories over an arbitrary time span.

In Appendix B, proofs are given for the equivalence of the quantum limit, outlined above, and the classical mechanics results in two cases: (I) $E_{0}>0$ with $\sigma=1$, and (II) $E_{0}<0$ with $\sigma=-1$. There are two further cases, each with opposite $\sigma$ to (I) and (II). They may be easily checked numerically. To this end, one starts with any proper curve parameter $w=w_{1}>0$ and determines the position $\xi=\xi\left(w_{1}\right)$, which by (84) or (85) delivers the classical time $\tau_{c l}=\tau(\xi)=\tau\left(\xi\left(w_{1}\right)\right)$. The corresponding quantum mechanical time $\tau_{q u}=\tau\left(w_{1}\right)$ is determined from (69) or (73).

\section{Summary}

For rectilinear motion, mean values are quantum mechanically derived in the macroscopic limit $\kappa=m X_{0} V_{0} / \hbar \rightarrow \infty$. The trajectories are described in terms of a curve parameter which is in 1-1 relation with time $t$. In the case of a headon collision, the theory predicts backscattering. If the mean energy is negative, the mass point oscillates between the target (force center) and a turning point; it stays on the same side of the force center always. For comparison, the 
classical trajectories are obtained from a one-dimensional Hamiltonian without regularization of the $(1 / r)$ potential. There appear branch points where the solutions are ambiguous. Within continuous intervals, which do not include the branch points, the equivalence of the quantum and the classical result is proved analytically.

Acknowledgements. The author expresses his gratitude to Jürgen Parisi for his constant encouragement and support. He also profited from his critical reading of the manuscript.

\section{A Calculation of mean values}

\section{A.1 Normalization condition}

$$
\begin{aligned}
1 & \equiv\left\langle\psi_{w} \mid \psi_{w}\right\rangle=\int_{0}^{2 \pi} \mathrm{d} \phi \int_{-\infty}^{\infty}\left(8 u^{2}\right) \mathrm{d} u_{1} \ldots \mathrm{d} u_{4} \Psi_{w}^{*}\left(\Phi^{\prime}\right) \Psi_{w}(\Phi) \\
& =-8 \pi^{2} C^{2} \int_{0}^{2 \pi} \mathrm{d} \phi \partial_{\Gamma}\left\{\left(1 / \Gamma^{2}\right) \exp [(\mathbf{A} \cdot \mathbf{A}) /(4 \Gamma)]\right\} \\
& =2 \pi^{2} C^{2} \int_{0}^{2 \pi} \mathrm{d} \phi\left\{\left(1 / \Gamma^{4}\right)[(\mathbf{A} \cdot \mathbf{A})+8 \Gamma] \exp [(\mathbf{A} \cdot \mathbf{A}) /(4 \Gamma)]\right\}_{\Gamma \rightarrow \Gamma_{R}} .
\end{aligned}
$$

The definition of $\mathbf{A}$, given by (14) and (15), implies that

$$
(\mathbf{A} \cdot \mathbf{A}) /\left(4 \Gamma_{R}\right)=K\left[\kappa_{0}+\kappa_{1} \cos (\phi)\right], \quad K=r_{0} \Gamma_{R}
$$

The $\phi$ integral is expressed in terms of the modified Bessel functions $I_{0}$ and $I_{1}$ as

$$
\int_{0}^{2 \pi} \mathrm{d} \phi \cos (\phi)^{n} \exp \left[K \kappa_{1} \cos (\phi)\right]=2 \pi I_{n}(\kappa), \quad n=0,1
$$

where we made use of the property that

$$
K \kappa_{1}=\kappa \equiv r_{0} \Gamma_{0}
$$

Eventually, we obtain

$$
1 \equiv 2\left(2 \pi r_{0} / K\right)^{3} C^{2} \exp \left(K \kappa_{0}\right) I_{0}(\kappa)\left[\kappa I_{\kappa}+2+K \gamma_{0} \sigma \sinh (2 w)\right]
$$

where $I_{\kappa}$ is defined in $(21)$ 


\section{A.2 Mean x component}

We use $x=2\left(u_{1} u_{3}-u_{2} u_{4}\right)$ and proceed in analogy to Appendix (B1) of [12].

$$
\begin{aligned}
\langle x\rangle & =C^{2} \int_{0}^{2 \pi} \mathrm{d} \phi \int_{-\infty}^{\infty}\left(8 u^{2}\right) \mathrm{d} u_{1} \ldots \mathrm{d} u_{4} 2\left(u_{1} u_{3}-u_{2} u_{4}\right) \exp \left[\mathbf{A} \cdot \mathbf{u}-\Gamma u^{2}\right] \\
& =-16 C^{2} \frac{\partial}{\partial \Gamma} \int_{0}^{2 \pi} \mathrm{d} \phi \int_{-\infty}^{\infty} \mathrm{d} u_{1} \ldots \mathrm{d} u_{4}\left[\frac{\partial^{2}}{\partial A_{1} \partial A_{3}}-\frac{\partial^{2}}{\partial A_{2} \partial A_{4}}\right] \exp \left[\mathbf{A} \cdot \mathbf{u}-\Gamma u^{2}\right] \\
& =-16 \pi^{2} C^{2} \frac{\partial}{\partial \Gamma} \int_{0}^{2 \pi} \mathrm{d} \phi\left[\frac{\partial^{2}}{\partial A_{1} \partial A_{3}}-\frac{\partial^{2}}{\partial A_{2} \partial A_{4}}\right] \frac{1}{\Gamma^{2}} \exp [\mathbf{A} \cdot \mathbf{A} /(4 \Gamma)]
\end{aligned}
$$

After carrying out the parameter differentiations and making us of the symmetry (16), we obtain

$$
\langle x\rangle=\frac{2 \pi^{2} C^{2}}{\Gamma_{R}^{6}} \int_{0}^{2 \pi} \mathrm{d} \phi A_{12}\left(A_{12}+8 \Gamma_{R}\right) \exp \left[A_{12} /\left(2 \Gamma_{R}\right)\right],
$$

where

$$
A_{12}=A_{1}^{2}+A_{2}^{2}=2 \Gamma_{R} K\left(\kappa_{0}+\kappa_{1} \cos (\phi)\right) .
$$

After setting $\Gamma_{R}=K / r_{0}$, we obtain

$$
\begin{aligned}
\langle x\rangle & =8 \pi^{2} r_{0}^{4} K^{-3} C^{2} \exp \left(K \kappa_{0}\right) \sum_{j=0}^{2} \int_{0}^{2 \pi} \mathrm{d} \phi c_{j} \cos ^{j}(\phi) \exp (\kappa \cos (\phi)) \\
& =\pi\left(4 \pi r_{0}^{2}\right)^{2} K^{-3} C^{2} \exp \left(K \kappa_{0}\right) I_{0}(\kappa)\left[c_{0}+c_{1} I_{\kappa}+c_{2}\left(-I_{\kappa} / \kappa+1\right)\right]
\end{aligned}
$$

which is the result (30) with the coefficients $c_{0}, c_{1}, c_{2}$ given in (31).

It is easily seen that the analogous integrands of $\langle y\rangle$ and $\langle z\rangle$ contain the factors $F_{y}$ and $F_{z}$ defined in (28) and, thus, vanish identically.

\section{A.3 Mean velocity}

According to [12], the velocity observable in $\mathbf{u}$ space reads

$$
v_{j}=\hbar /(2 \mathbf{i} m) D_{j}, \quad j=x, y, z,
$$

with

$$
\begin{aligned}
D_{x} & =\left(1 / u^{2}\right)\left[u_{3} \partial_{u_{1}}-u_{4} \partial_{u_{2}}+u_{1} \partial_{u_{3}}-u_{2} \partial_{u_{4}}\right], \\
D_{y} & =\left(1 / u^{2}\right)\left[u_{4} \partial_{u_{1}}+u_{3} \partial_{u_{2}}+u_{2} \partial_{u_{3}}+u_{1} \partial_{u_{4}}\right], \\
D_{z} & =\left(1 / u^{2}\right)\left[u_{1} \partial_{u_{1}}+u_{2} \partial_{u_{2}}-u_{3} \partial_{u_{3}}-u_{4} \partial_{u_{4}}\right] .
\end{aligned}
$$


From Appendix (C1) of [12], we take over the mean value integral in slightly modified form,

$$
\begin{aligned}
\left\langle D_{x}\right\rangle= & 4 C^{2} \int_{0}^{2 \pi} \mathrm{d} \phi \int_{-\infty}^{\infty} \mathrm{d} u_{1} \mathrm{~d} u_{2} \mathrm{~d} u_{3} \mathrm{~d} u_{4} \exp \left[\mathbf{a}^{*}(\Phi) \cdot \mathbf{u}-\Gamma^{*} u^{2} / 2\right] \times \\
& \left\{\left[u_{3} \partial_{u_{1}}-u_{4} \partial_{u_{2}}+u_{1} \partial_{u_{3}}-u_{2} \partial_{u_{4}}\right] \exp \left[\mathbf{a}\left(\Phi^{\prime}\right) \cdot \mathbf{u}-\Gamma u^{2} / 2\right]\right\} \\
= & -4 C^{2} \int_{0}^{2 \pi} \mathrm{d} \phi \int_{-\infty}^{\infty} \mathrm{d} u_{1} \mathrm{~d} u_{2} \mathrm{~d} u_{3} \mathrm{~d} u_{4}\left[u_{3}\left(a_{1}-\Gamma u_{1}\right)-u_{4}\left(a_{2}-\Gamma u_{2}\right)+\right. \\
& \left.u_{1}\left(a_{3}-\Gamma u_{3}\right)-u_{2}\left(a_{4}-\Gamma u_{4}\right)\right] \exp \left[\mathbf{A} \cdot \mathbf{u}-\Gamma_{R} u^{2}\right]
\end{aligned}
$$

It is observed that the metric factor $u^{2}$ cancels out against the $\left(1 / u^{2}\right)$ factor of the operator $D_{x}$. After generating the polynomial $u_{i}$ factors by parameter differentiation with respect to $A_{i}$, the Gaussian $u_{i}$ integrals are performed. Analogous expressions are obtained for $\left\langle D_{y}\right\rangle$ and $\left\langle D_{z}\right\rangle$. Intermediate results are stated in (36) and (37). The evaluation of $\left\langle v_{x}\right\rangle$ proceeds straightforwardly to give the result (39) with (40).

\section{A.4 Mean reciprocal distance}

Since $1 / r=1 / u^{2}$, the metric factor $u^{2}$ cancels out; furthermore, there are no polynomial $u_{i}$ factors to be generated by parameter differentiation. One obtains straightforwardly the results stated in Sec.III. B.

\section{B Proof for the equivalence of leading order quantum and of classical mechanics}

In the following, we prove analytically the equality of the classical and quantum mechanical result (of leading order in $\kappa$ ) for the time $\tau$ in two cases: (I) $E_{0}>0$ with $\sigma=1$ and (II) $E_{0}<0$ with $\sigma=-1$.

\section{B.1 Case (I)}

We compare the result (69) for $\tau=\tau(w)$ with the result (84) for $\tau=\tau(\xi)$. The basic definition interval of (84) for $\sigma=1$ is $\xi \geq 1$ which corresponds to the interval of the curve parameter $w \geq 0$. In order to invert the function $\xi(w)$ for $w$, see $(66 \mathrm{a})$, we take the square root and substitute $(1 / 2)(u+1 / u)$ for $\cosh (w)$ and $(1 / 2)(u-1 / u)$ for $\sinh (w)$ with $u=\exp (w)$. We solve the following equation for $u$ :

$$
\sqrt{\xi}=(1 / 2)[u+1 / u+\lambda(u-1 / u)]
$$


with the result

$$
u=\frac{\sqrt{\xi}+\sqrt{\lambda^{2}-1+\xi}}{1+\lambda}, \quad w=\ln (u) .
$$

When (69) is compared with (84), it is observed that the first term on the right hand side of (69) and the second term of the right hand side of (84) correspond and by (99) are equal to one another:

$$
w\left(1-\lambda^{2}\right) \equiv\left(1-\lambda^{2}\right) \ln \left[\left(\sqrt{\xi}+\sqrt{\lambda^{2}-1+\xi}\right) /(1+\lambda)\right],
$$

where we used the relation $\mu=1-\lambda^{2}$. The following identity remains to be shown in the intervals $\xi \geq 1, w \geq 0, \sigma=1$, and $\lambda>1$ :

$$
2 \lambda \sinh (w)^{2}+\left(1+\lambda^{2}\right) \cosh (w) \sinh (w) \equiv \sqrt{\xi\left(\lambda^{2}-1+\xi\right)}-\lambda .
$$

This can be seen, for instance, by squaring both sides of (101) and by inserting on the left hand side $(1 / 2)(u+1 / u)$ for $\cosh (w)$ and $(1 / 2)(u-1 / u)$ for $\sinh (w)$, and eventually using (99) to express $u$ by $\xi$.

\section{B.2 Case (II)}

For $\sigma=-1$, we have to compare the result (73) with $\tau=\tau(\tilde{w})$ and (85) with $\tau=\tau(\xi)$. The definition domains of (85) are $0<\xi \leq 1$ with $0 \leq \tilde{w}<\pi / 2$, where the latter inequality is due to the fact that at $\xi=0$ the velocity gets infinite which by $(67 \mathrm{~b})$ occurs if $\tan (\tilde{w})=1 / \tilde{\lambda}$.

We invert $\xi(\tilde{w})$ for $\tilde{w}$. By (67a), we solve the equation

$$
0=\sqrt{\xi}-\left[\sqrt{1-S^{2}}-\tilde{\lambda} S\right], \quad S=\sin (\tilde{w}),
$$

for $S$, and obtain

$$
\begin{aligned}
& \sin (\tilde{w}) \equiv S=\left[\sqrt{1+\tilde{\lambda}^{2}-\xi}-\tilde{\lambda} \sqrt{\xi}\right]\left[1+\tilde{\lambda}^{2}\right]^{-1}, \\
& \cos (\tilde{w}) \equiv \sqrt{1-S^{2}}=\left[\tilde{\lambda} \sqrt{1+\tilde{\lambda}^{2}-\xi}+\sqrt{\xi}\right]\left[1+\tilde{\lambda}^{2}\right]^{-1} .
\end{aligned}
$$

Using $\tan (\tilde{w})=\sin (\tilde{w}) / \cos (\tilde{w})$, we derive with the aid of (104)

$$
\tan (\tilde{w}) \equiv A=\left[\sqrt{1+\tilde{\lambda}^{2}-\xi}-\tilde{\lambda} \sqrt{\xi}\right]\left[\tilde{\lambda} \sqrt{1+\tilde{\lambda}^{2}-\xi}+\sqrt{\xi}\right]^{-1} .
$$

The following terms of (73) and (85) are identical for $\sigma=-1$ and $\mu=-\left(1+\tilde{\lambda}^{2}\right)$ :

$$
\tilde{w}\left(1+\tilde{\lambda}^{2}\right) \equiv-\left(1+\tilde{\lambda}^{2}\right)\left[\arctan \left(\sqrt{\frac{\xi}{-\xi-\mu}}\right)-\arctan \left(\frac{1}{\tilde{\lambda}}\right)\right] .
$$


For verification, one inserts on the left hand side $\arctan (A)$ according to (104); furthermore, one applies the addition formula $\arctan \left(a_{1}\right)+\arctan \left(a_{2}\right)=$ $\arctan \left(\left(a_{1}+a_{2}\right) /\left(1-a_{1} a_{2}\right)\right)$. The remaining terms of (73) and (85) have to obey the identity

$$
\left(1-\tilde{\lambda}^{2}\right) \cos (w) \sin (w)-2 \tilde{\lambda} \sin (w)^{2} \equiv \sqrt{\xi\left(1+\tilde{\lambda}^{2}-\xi\right)}-\tilde{\lambda}
$$

On the left hand side, one substitutes the results of (103) for the trigonometric functions and obtains the right hand side after elementary rearrangements.

\section{References}

[1] M. Abramowitz and I. Segun, Handbook of Mathematical Functions, Dover Publications, New York, 1968.

[2] C. Chen and M. Kibler, Connection between the hydrogen atom and the four-dimensional oscillator, Phys. Rev. A, 31 (1985), 3960-3963.

https://doi.org/10.1103/physreva.31.3960

[3] S. Coussy, S. Grangeon, Ph. Bataillard, Hicham Khodja, Nicolas Maubec, Pierre Faure, Christophe Schwartz, Robin Dagois, Evolution of iron minerals in a 100 years-old technosol. Consequences on Zn mobility, Geoderma, 290 (2017), 19-32. https://doi.org/10.1016/j.geoderma.2016.12.009

[4] H. Goldstein, Klassische Mechanik, Akademische Verlagsgesellschaft, Frankfurt, 1963.

[5] T.T. Hlatshwago, L.D. Sebitla, E.G. Njoroge, M. Mlambo, J.B. Malherbe, Annealing effects on the migration of ion-implanted cadmium in glassy carbon, Nucl. Instrum. Methods Phys. Res. Sect. B, 395 (2017), 34-38. https://doi.org/10.1016/j.nimb.2017.01.086

[6] C. Jaeger, T. Sabri, E. Wendler, Th. Henning, Ion-induced processing of cosmic silicates: a possible formation pathway of gems, Astrophys. J., 831 (2016), 66. https://doi.org/10.3847/0004-637x/831/1/66

[7] P. Kustaanheimo and E. Stiefel, Perturbation theory of Kepler motion based on spinor regularization, J. Reine Angew. Math., 1965 (1965), no. 218, 204. https://doi.org/10.1515/crll.1965.218.204

[8] M. McCall, Gravitational orbits in one dimension, Am. J. Phys., 74 (2006), 1115-1119. https://doi.org/10.1119/1.2348890 
[9] T. Pradell, G. Molina, J. Molera, M. S. Tite, Composition of the lustre pigment in the production of 13th century AD Raqqa lustreware from Syria, Archaeometry, 58 (2016), 979-986.

https://doi.org/10.1111/arcm.12211

[10] K. Oura, V.G. Lifshits, A. A. Saranin, A. V. Zotov and M. Katayama, Surface Science: An Introduction, Springer-Verlag, Berlin, 2003.

https://doi.org/10.1007/978-3-662-05179-5

[11] A. Rauh and J. Parisi, Quantum mechanics of hyperbolic orbits in the Kepler problem, Phys. Rev. A, 83 (2011), 042101. https://doi.org/10.1103/physreva.83.042101

[12] A. Rauh and J. Parisi, Quantum mechanics of Kepler orbits, Adv. Stud. in Theor. Phys., 8 (2014), 889-938.

https://doi.org/10.12988/astp.2014.48114

[13] A. Rauh and J. Parisi, Quantum mechanical correction to Kepler's equation, Adv. Stud. in Theor. Phys., 10 (2016), 1-22.

https://doi.org/10.12988/astp.2016.510105

[14] A. Rauh, Coherent states of harmonic and reversed harmonic oscillator, Symmetry, 8 (2016), no. 6, 46. https://doi.org/10.3390/sym8060046

[15] S. Wolfram, Mathematica Version 10.1.0.0, Wolfram Research, Champaign, IL., USA, 2015.

[16] S. Zang, K. Nordlund, F. Djurabekova, Y. Zhang, G. Velisa, T. S. Wang, Simulation of Rutherford backscattering spectrometry from arbitrary atom structures, Phys. Rev. E, 94 (2016), 04331.

https://doi.org/10.1103/physreve.94.043319

[17] To be uploaded to the author's account at ResearchGate after acceptance for publication.

Received: May 19, 2017; Published: June 23, 2017 